\title{
Predominant influence of MGMT methylation in non-resectable glioblastoma after radiotherapy plus temozolomide
}

\author{
Niklas Thon, ${ }^{1}$ Sabina Eigenbrod, ${ }^{2}$ Eva M Grasbon-Frodl, ${ }^{2}$ Juergen Lutz, ${ }^{3}$ \\ Simone Kreth, ${ }^{4}$ Gabriele Popperl, ${ }^{5}$ Claus Belka, ${ }^{6}$ Hans A Kretzschmar, ${ }^{2}$ \\ Joerg-Christian Tonn, ${ }^{1}$ Friedrich W Kreth ${ }^{1}$
}

${ }^{1}$ Department of Neurosurgery, University Hospital Großhadern, Ludwig-Maximilians-Universität München, Munich, Germany ${ }^{2}$ Center for Neuropathology and Prion Research, University Hospital Großhadern, Ludwig-Maximilians-Universität München, Munich, Germany ${ }^{3}$ Department of Clinical Radiology, University Hospital Großhadern,

Ludwig-Maximilians-Universität München, Munich, Germany ${ }^{4}$ Department of Anaesthesiology, University Hospital Großhadern, Ludwig-Maximilians-Universität München, Munich, Germany ${ }^{5}$ Department of Nuclear Medicine, University Hospital Großhadern,

Ludwig-Maximilians-Universität München, Munich, Germany ${ }^{6}$ Department of Radiation Oncology, University Hospital Großhadern,

Ludwig-Maximilians-Universität München, Munich, Germany

\section{Correspondence to}

Professor Dr Friedrich W Kreth, Department of Neurosurgery, Ludwig-Maximilians-Universität München, Marchioninistr. 15, 81377 Munich, Germany; fkreth@med.uni-muenchen.de

Parts of the study have been presented as an oral presentation at the SNO's 13th Annual Scientific Meeting, Lake Las Vegas Resort, 22-23 November 2008, and as a poster presentation at the 2009 AACR Annual Meeting. 18-22 April 2009, Denver, Colorado.

Received 16 April 2010 Revised 25 June 2010 Accepted 7 July 2010 Published Online First 22 September 2010

\section{ABSTRACT}

Background Patients with non-resectable glioblastoma generally exhibit a poor prognosis, even after radiotherapy plus concomitant and adjuvant temozolomide (XRT/TMZ $\rightarrow$ TMZ). Unfortunately, no data are available concerning the predictive value of $0^{6}$-methylguanine-DNA methyltransferase (MGMT) promoter methylation for this important subpopulation. For clarification, a prospective study was conducted.

Methods Adult patients with a non-resectable glioblastoma were included. A molecular stereotactic biopsy technique was used for tumour characterisation combining histopathological diagnosis with small sample size adjusted methylation-specific PCR (MSP) and sodium bisulfite sequencing. Treatment included XRT (60 Gy in 30 fractions)/TMZ (daily dose of $75 \mathrm{mg} / \mathrm{m}^{2}$ ) $\rightarrow$ TMZ (150-200 mg/m² per day for 5 days of every 28-day cycle). The primary end point was progression-free survival (PFS). Secondary endpoints were overall survival (OS) and treatment response (TR). Patients were categorised in the Radiation Therapy Oncology Group (RTOG)-recursive partitioning analysis (RPA) Classes III ( $\mathrm{N}=4)$, IV ( $=12), \mathrm{V}(\mathrm{N}=28)$ and $\mathrm{VI}$ $(\mathrm{N}=12)$.

Results and discussion The success rates of MSP and sequence analyses were 100\%. The MGMT promoter was methylated in 30/56 tumours, which was associated with an increased PFS (median 56 versus 20 weeks; hazard ratio 0.15 ; range 0.07 to 0.33 ; $p<0.0001)$, higher frequency of TR $(93.3 \%$ vs $46.2 \%$; $\mathrm{p}=0.0008$ ) and increased OS (median 104 vs 28 weeks; hazard ratio 0.18 ; range 0.08 to $0.38 ; p<0.0001$ ). The transient perioperative morbidity was $1.8 \%$.

Conclusion MGMT promoter methylation has a predominant favourable influence even for the important subpopulation with non-resectable glioblastoma. The molecular stereotactic biopsy technique is safe and effective for predictive evaluation and helps to avoid both over- and undertreatment.

\section{INTRODUCTION}

The incidence of malignant gliomas has increased over the last two decades, especially in the older subpopulation, and currently lies in the range of five cases per $100000 .^{1}$ Glioblastoma account for approximately $60-70 \%$ of these tumours. Recently, important progress has been achieved in the treatment of this most malignant tumour. First, radiotherapy plus concomitant and adjuvant temozolomide (XRT/TMZ $\rightarrow \mathrm{TMZ}$ ) has resulted in significant prolongation of both time to progression and survival as compared with XRT alone; this was demonstrated by the results of the prospective randomised trial performed by the European Organisation for Research and Treatment of Cancer (EORTC) and the National Cancer Institute of Canada (NCIC) Clinical Trials Group. ${ }^{3}{ }^{3}$ Second, epigenetic silencing of the promoter of the gene that encodes $\mathrm{O}^{6}$-methylguanine-DNA methyltransferase (MGMT) in tumour specimens has been identified as a favourable predictive factor of benefit from XRT/TMZ $\rightarrow \mathrm{TMZ}$; this was shown in a companion retrospective study. ${ }^{4}$

The impact of these findings on clinical practice, however, is still under debate: subgroup analysis suggests that relative young and/or fit patients harbouring a tumour with a methylated MGMT promoter and undergoing resection rather than biopsy benefit most from XRT/TMZ $\rightarrow \mathrm{TMZ}^{2}{ }^{2}$ It remains unclear as to which extent unfavourable prognostic factors such as increased age and/or a deep seated tumour location might outweigh potential benefit resulting from MGMT methylation. ${ }^{5}$ The important subgroup of patients with non-resectable glioblastoma is particularly concerned: patients having undergone biopsy only experienced the worst outcome in the EORTC/NCIC trial, which was irrespective of treatment ${ }^{2}$ - however, no stratification by the MGMT methylation status was done, because this biomarker could not be analysed from small biopsy specimens. ${ }^{4}$ In this situation, oncologists are confronted with unawareness and uncertainty concerning appropriate treatment strategies for those patients not suitable for open tumour resection. The current prospective study was conducted to elucidate for the first time the prognosis of patients with non-resectable glioblastoma undergoing XRT/TMZ $\rightarrow \mathrm{TMZ}$ after adjustment for the effects of $M G M T$ promoter methylation and other relevant prognostic factors. Characterisation of the tumour was performed using the molecular stereotactic biopsy technique which combines state-of-theart histopathological evaluation with a small sample size adjusted molecular genetic analysis. ${ }^{6-8}$ The current study should not be regarded as an appendix to the EORTC/NCIC trial. Both studies differ in terms of selection criteria and applied treatment strategies. 


\section{METHODS \\ Patients}

Adult patients (aged 18-85 years) were eligible if they had (1) a supratentorial glioblastoma not suitable for gross total tumour resection (because of an eloquent tumour location and/or significant comorbidity) with histology being proven by stereotactic biopsy, (2) no severe mass effect of the tumour demanding debulking surgery because of brainstem compression and/or midline shift, (3) no prior history of surgery, XRT and/or chemotherapy, (4) a Karnofsky performance score (KPS) $\geq 60^{9}$ and (5) adequate haematologic, renal and hepatic function (as being defined before). ${ }^{3}$ All enrolled patients gave informed consent. The study protocol was approved by the institutional review board (AZ 216/14) of the Ludwig Maximilians University Munich, Germany.

\section{Molecular stereotactic biopsy technique}

The still new technique of molecular stereotactic biopsy has been described previously. ${ }^{6-8}$ In brief, coregistration of computerised tomography (CT) and magnetic resonance imaging (MRI; including T1- and T2-weighted sequences) served for three-dimensional (3D) visualisation (i-plan stereotaxy, BrainLAB, Feldkirchen, Germany) of the tumour and the simulation of the best trajectory. Serial biopsies were taken along a trajectory representative of the solid tumour. Using microforceps, the maximum amount of tissue per biopsy specimen was $1 \mathrm{~mm}^{3}$. The number of specimens taken in $1 \mathrm{~mm}$ steps along the chosen trajectory was in the range of 10-18 samples per tumour. The tissue-sampling procedure was guided by intraoperative smear preparations, which were routinely performed by the attending neuropathologist: only tumour probes next (ie, $1 \mathrm{~mm}$ distance) to smear preparations exclusively showing solid vital tumour tissue were used for molecular genetic analysis; a corresponding sample (level $+1 \mathrm{~mm}$ ) was taken for paraffin embedding and histopathological examination using standard protocols. ${ }^{8}$ Samples chosen for molecular genetic analysis were snap-frozen immediately after withdrawal. The described biopsy technique was chosen to minimise the risk of tissue contamination (eg, by nonneoplastic or necrotic tissue) and, more importantly, to recognise contamination, if it occurs. For determination of the MGMT promoter methylation status, at least two tissue samples were collected from different sites throughout each tumour to test for both the intratumoural distribution of the biomarker and the reproducibility of the molecular genetic analysis. The histological diagnosis of all tissue specimens was made according to the 2007 World Health Organization (WHO) classification of tumours of the central nervous system. ${ }^{10} \mathrm{~A}$ CT-scan was done 1 day after biopsy in all patients, and any sequels attributed to the biopsy procedure were classified as morbidity. ${ }^{11}$

\section{Methylation-specific PCR (and sequencing analysis)}

Isolation of nucleic acids, bisulfite modification of DNA, methylation-specific PCR (MSP) and sequencing analyses were done as published previously in detail. ${ }^{812}$ In brief, DNA isolation from tumour specimens was performed using the OIAamp DNA Micro Kit (Qiagen, Hilden, Germany), and DNA recovery from each stereotactic biopsy sample amounted to around 30-60 ng/ $\mu 1$. This step was followed by purification and bisulfite modification of DNA according to Mollemann et al. ${ }^{12}$ MSP and sequencing analyses were performed using the specific primer pairs as described previously. ${ }^{13-15}$ 'Unmethylated' and 'methylated' tumours were defined according to Grasbon-Frodl et al. ${ }^{8}$
Sequencing of bisulfite-modified DNA indicated a methylated promoter when more than half of the CpG sites (13 of $25 \mathrm{CpG}$ sites) were found to be methylated; partial methylation was defined as the cytosine and thymine peaks being equally sized or the cytosine peak being twice as high as the corresponding thymine peak.

\section{Study design}

Patients were enrolled in the period from March 2006 to August 2008. Patients were categorised according to the Radiation Therapy Oncology Group (RTOG)-recursive partitioning analysis (RPA) classes. ${ }^{16}$ The KPS was used for clinical evaluation. ${ }^{9}$ Rating on the KPS was done independently by two investigators (NT and FWK). In case of discordantly rated performance scores, a third investigator (JCT) was introduced, and the most matching score was used thereafter. Histopathological diagnosis and determination of the MGMT methylation status were obtained within 5-7 working days. Within 3 weeks after stereotactic biopsy, patients were assigned to receive XRT/TMZ $\rightarrow \mathrm{TMZ}$ : treatment included XRT (60 grey in 30 fractions)/TMZ [daily dose of $75 \mathrm{mg} / \mathrm{m}^{2}$ ] $\rightarrow \mathrm{TMZ}$ $\left(150-200 \mathrm{mg} / \mathrm{m}^{2}\right.$ per day for 5 days of every 28 -day cycle). In case of long-term compliance, however, TMZ was continued (at the same dose) until tumour progression occurred. At baseline evaluation, 4-6 weeks after XRT/TMZ and every three cycles during TMZ maintenance therapy, clinical and neuroradiological examinations were performed (until last follow-up). MRI interpretation was independently done according to the Macdonald criteria ${ }^{17}$ by an experienced neuroradiologist (JL), who was blinded for the MGMT status and the follow-up data of the patient. Treatment response was evaluated after the completion of three TMZ cycles or earlier in case of clinical deterioration. Tumour progression had to be confirmed by further clinical and neuroradiological follow-up to exclude any bias by pseudoprogression. ${ }^{18}$ Additional metabolic imaging ${ }^{19}$ and/or stereotactic rebiopsy were considered necessary, when further (modified) invasive treatment strategies were under consideration. Haematology was performed weekly. Adverse events were defined according to the National Cancer Institute (NCI) Common Toxicity Criteria, version 3.0. The minimum follow-up after inclusion of the last patient had to be 6 months.

\section{Statistical analysis}

The hazard ratio for tumour progression was in the range of 0.48 in favour of the MGMT promoter methylated group in the study of Hegi et al. ${ }^{4}$ In the absence of cytoreductive surgery, we expected an even more pronounced outcome difference; this assumption was indirectly supported by the study of Chinot et $a l^{20}$ who have found highly divergent outcome scores as a function of MGMT expression after neoadjuvant TMZ of non-resectable glioblastoma (median progression-free survival (PFS): 5.5 vs 1.9 months). In the current series, a hazard ratio of 0.4 or even less in favour of the MGMT promoter methylated group was expected. Assuming exponential survival, we estimated that a sample size in the range of 27 patients or more in each group would be sufficient to have a power of $80 \%$ to demonstrate a significant difference in PFS in favour of glioblastoma with a methylated MGMT promoter.

The reference point of this study was the date of the diagnosis. The primary endpoint was PFS; secondary endpoints were treatment response (TR) and overall survival (OS). PFS and OS were analysed by the Kaplan-Meier method ${ }^{21}$ and compared with the two-sided logrank test. Logistic regression 
models were used to analyse the association between TR and other variables. The Cox model was fitted to assess the prognostic value of the MGMT methylation status and other potential prognostic factors. ${ }^{22}$ First, the importance of each variable was tested univariately. Second, all variables were fitted together. The 'BEST' model contained only variables associated with PFS or OS after adjustment for the effects of the other variables. The distribution of patient- and tumourrelated variables between $M G M T$ promoter methylated and unmethylated subgroups was analysed by the $\chi^{2}$ statistics (for dichotomised variables) and the Wilcoxon test (for continuously scaled variables). A p value of $\leq 0.05$ was considered significant. All calculations were performed using the SAS software package (version 9.1).

\section{RESULTS}

\section{Patient characteristics}

Out of an overall number of 136 patients newly diagnosed as having a glioblastoma in our department between March 2006 and August 2008, a total of 56 consecutive patients (33 men, 23 women) fulfilled inclusion criteria and were enrolled in this study. Patients were categorised into RTOG-RPA class III (four patients, $7.1 \%)$, class IV (12 patients, $21.4 \%)$, class V (28 patients, $50.0 \%$ ) and class VI (12 patients, $21.4 \%)$. The median of the age distribution was 62.5 years (range 23-85 years); 22 $(39 \%)$ patients were 65 years of age or older. The median KPS was 70; 24 patients (42.9\%) experienced a KPS of 70, and 13 a KPS of 60 (23.2\%). Forty-eight patients (85.7\%) exhibited multifocal (six patients) or eloquent (42 patients) tumour locations including involvement of the primary motor/sensory cortex (10 patients), speech area (eight patients), visual cortex (three patients), basal ganglia (16 patients) and the corpus callosum (five patients). Stereotactic biopsy procedures were associated with a transient morbidity of $1.8 \%$ (one patient with transient aphasia). MSP and sequencing analyses always exhibited concordant and reproducible results throughout each tumour (mean number of samples per tumour: 3; range 2-5). The overall frequency of MGMT methylated tumours was $53.6 \%(30 / 56)$ including one patient with a partially methylated promoter. Methylated and unmethylated subpopulations did not differ with respect to patients' characteristics, even though patients with a methylated MGMT promoter were somewhat younger (table 1).

One patient was lost to follow-up 24 weeks after biopsy without experiencing tumour progression at this time. Two patients had to be discontinued from concomitant TMZ after 3 and 4 weeks, respectively, because of grade 3 haematological toxicity and lung embolism. Adjuvant TMZ was not initiated in three patients because of low clinical performance $(\mathrm{KPS}<60)$ after XRT/TMZ, which was associated with a decline in the mental status (disorientation and confusion). TMZ maintenance therapy caused grade $1 / 2$ toxicity in 12 patients. As a consequence of grade 3 toxicity, adjuvant TMZ had to be interrupted for 4 weeks in two patients and to be discontinued after two cycles in one patient (table 2).

Adverse events were more often seen in older patients (median age of patients with/without adverse events: 70 versus 57.5 years; $p<0.03)$. The application of more than six TMZ cycles (15 patients with a median number of nine TMZ cycles) was not associated with increased toxicity. However, late grade 2 haematotoxicity was seen in two patients after the 11th and 14th cycle of TMZ, respectively. MRI-based suspicion of tumour progression was additionally verified by metabolic imaging
Table 1 Characteristics and clinical outcome of patients with glioblastoma according to the methylation status of the MGMT promoter

\begin{tabular}{|c|c|c|c|c|}
\hline & & $\begin{array}{l}\text { Unmethylated } \\
\text { (N=26) }\end{array}$ & $\begin{array}{l}\text { Methmethylated } \\
(\mathrm{N}=30)\end{array}$ & p Value \\
\hline \multicolumn{5}{|l|}{ Characteristics } \\
\hline \multirow[t]{4}{*}{ Age (years) } & Median & 64.5 & 58.5 & NS \\
\hline & $<50$ & $7(26.9 \%)$ & $10(33.3 \%)$ & \\
\hline & $50-60$ & $3(11.6 \%)$ & $6(20.0 \%)$ & \\
\hline & $>60$ & $16(61.6 \%)$ & $14(46.7 \%)$ & \\
\hline $\begin{array}{l}\text { Karnofsky performance } \\
\text { score }\end{array}$ & Median & 70 & 70 & NS \\
\hline \multirow{4}{*}{$\begin{array}{l}\text { Radiation Therapy } \\
\text { Oncology Group- } \\
\text { recursive partitioning } \\
\text { analysis }\end{array}$} & III & $1(3.8 \%)$ & $3(10.0 \%)$ & \\
\hline & IV & $6(23.1 \%)$ & $6(20.0 \%)$ & \\
\hline & $\mathrm{V}$ & $12(46.2 \%)$ & 16 (53.3\%) & \\
\hline & $\mathrm{VI}$ & 7 (26.9\%) & $5(16.7 \%)$ & \\
\hline \multirow[t]{2}{*}{ Sex } & Male & $15(57.7 \%)$ & $18(60.0 \%)$ & NS \\
\hline & Female & $11(42.3 \%)$ & $12(40.0 \%)$ & \\
\hline Tumour volume (ml) & Mean (SD) & $43.7( \pm 24.9)$ & $38.8( \pm 31.2)$ & \\
\hline \multirow[t]{2}{*}{ Tumour side } & Right & $10(38.5 \%)$ & $14(46.7 \%)$ & NS \\
\hline & Left & $16(61.5 \%)$ & $16(53.3 \%)$ & \\
\hline \multirow[t]{3}{*}{ Tumour location } & Lobar & $3(11.5 \%)$ & $5(16.7 \%)$ & NS \\
\hline & Eloquent & $21(80.8 \%)$ & $21(70.0 \%)$ & \\
\hline & Multifocal & $2(7.8 \%)$ & $4(13.3 \%)$ & \\
\hline \multicolumn{5}{|l|}{ Outcome } \\
\hline \multirow[t]{4}{*}{ Treatment response } & & & & 0.0008 \\
\hline & $\begin{array}{l}\text { Partial } \\
\text { response }\end{array}$ & $3(11.6 \%)$ & $17(56.7 \%)$ & \\
\hline & $\begin{array}{l}\text { Stable } \\
\text { disease }\end{array}$ & $9(34.6 \%)$ & $11(36.6 \%)$ & \\
\hline & Progression & $14(53.8 \%)$ & $2(6.7 \%)$ & \\
\hline \multirow[t]{5}{*}{ Progression-free surviva } & & & & $<0.0001$ \\
\hline & $\begin{array}{l}\text { Median } \\
\text { (weeks) }\end{array}$ & 20 & 56 & \\
\hline & $\begin{array}{l}\text { Rate at } \\
6 \text { months }\end{array}$ & $34.6 \%$ & $83.3 \%$ & \\
\hline & $\begin{array}{l}\text { Rate at } \\
12 \text { months }\end{array}$ & $0 \%$ & $54.4 \%$ & \\
\hline & $\begin{array}{l}\text { Hazard ratio } \\
(95 \% \mathrm{Cl})\end{array}$ & & $0.15(0.07$ to 0.33$)$ & \\
\hline \multirow[t]{5}{*}{ Overall survival } & & & & $<0.0001$ \\
\hline & $\begin{array}{l}\text { Median } \\
\text { (weeks) }\end{array}$ & 28 & 104 & \\
\hline & $\begin{array}{l}\text { Rate at } \\
6 \text { months }\end{array}$ & $57.7 \%$ & $90.0 \%$ & \\
\hline & $\begin{array}{l}\text { Rate at } \\
12 \text { months }\end{array}$ & $13.2 \%$ & $64.8 \%$ & \\
\hline & $\begin{array}{l}\text { Hazard ratio } \\
(95 \% \text { Cl) }\end{array}$ & & $0.18(0.08$ to 0.38$)$ & \\
\hline
\end{tabular}

NS, not significant.

[O-(2-[18F]fluoroethyl)-L-tyrosine (FET) positron emission tomography (PET)] in 20 patients and/or rebiopsy in 13 patients; the latter always revealed an unchanged MGMT promoter methylation status.

Table 2 Toxicity symptoms*

\begin{tabular}{lll}
\hline & No of patients (\%) (N=56) & \\
\hline $\begin{array}{l}\text { National Institute of Cancer Common } \\
\text { Toxicity Criteria Grade }\end{array}$ & $1-2$ & $3-4$ \\
Myelosuppression & $4(7)$ & $3(5.4)$ \\
Thrombosis & $\mathrm{MV}$ & $4(7)$ \\
Nausea & $9(16)$ & $\mathrm{MV}$ \\
Infection & $5(9)$ & $\mathrm{MV}$ \\
Mental status $\dagger$ & $7(13)$ & $7(13)$ \\
Otherf & $4(7)$ & $1(2)$ \\
\hline
\end{tabular}

*One patient can suffer from multiple symptoms.

†Including cognitive impairment, disorientation, confusion, depression and psychosis.

łIncluding hypocortisolism, apoplex, cardial dysrhythmias and pruritus. 


\section{Clinical outcome}

The median follow-up time was 48 weeks (range 24-144) for the survivors. Forty-one out of 56 patients exhibited tumour progression, and 34 patients had died. Death was tumour-related in all patients. Kaplan-Meier estimates for PFS and OS of the whole study population are presented in figure 1. Both primary and secondary end points were in favour of MGMT promoter methylated tumours (table 1): MGMT promoter methylated tumours exhibited superior median PFS (56 vs 20 weeks) and superior median OS (104 vs 28 weeks); PFS after 6 (12) months was $83.3 \%(54.4 \%)$ for MGMT promoter methylated tumours and $34.6 \%(0 \%)$ for those with an unmethylated MGMT promoter $(\mathrm{p}<0.0001)$ (figure 2$)$. The corresponding 6 (12) months survival rates were $90.0 \%(64.8 \%)$ and $57.7 \%(13.2 \%)$, respectively $(p<0.0001)$ (figure 2$)$. Among patients with $M G M T$ promoter methylation, the unadjusted hazard ratio for disease progression and death was 0.15 (95\% CI 0.07 to 0.33 ) and 0.18 ( $95 \%$ CI 0.08 to 0.38 ), respectively.

Treatment responders showing partial response or stable disease ( 3 months after treatment) were more often seen in case of a methylated MGMT promoter (28/30 MGMT methylated tumours vs $12 / 26$ unmethylated tumours; $p=0.0008)$. Treatment responders were younger and experienced a longer $O S$ than non-responders (median: 72 vs 18 weeks; $\mathrm{p}<0.0001$ ) (see table 3 ).

The survival advantage, however, was less pronounced for those with an unmethylated MGMT promoter (median: 44 weeks vs 104 weeks, $\mathrm{p}<0.001)$. At the time of tumour progression, treatment concepts included second-line chemotherapy $(n=16)$, reirradiation $(n=4)$, tumour debulking due to significant space occupying effects $(n=2)$, stereotactic brachytherapy $(n=3)$, a combination of these treatment modalities $(n=8)$ and palliative care $(n=23)$. The median survival after progression was longer for methylated tumours (3.7 months versus 1.9 months); the difference, however, was not significant.

\section{Prognostic and predictive factors}

Univariately, MGMT promoter methylation, younger age, higher KPS scores and RTOG-RPA classes III+IV were positively correlated with both increased PFS and OS. In the 'BEST' prognostic Cox models, only MGMT promoter methylation status (OS: $p<0.0001$; PFS: $p<0.0001$ ), younger age (PFS: $\mathrm{p}=0.02)$ and higher KPS scores (OS: $p=0.02)$ remained statistically significant (table 4).

The adjusted hazard ratio of 0.2 (95\% CI 0.09 to 0.43 ) of MGMT promoter methylation for PFS was consistent with the unadjusted hazard ratio of 0.15 ( $95 \%$ CI 0.07 to 0.33 ). Logistic regression analysis identified MGMT promoter methylation as the only predictor for TR (partial response or stable disease;

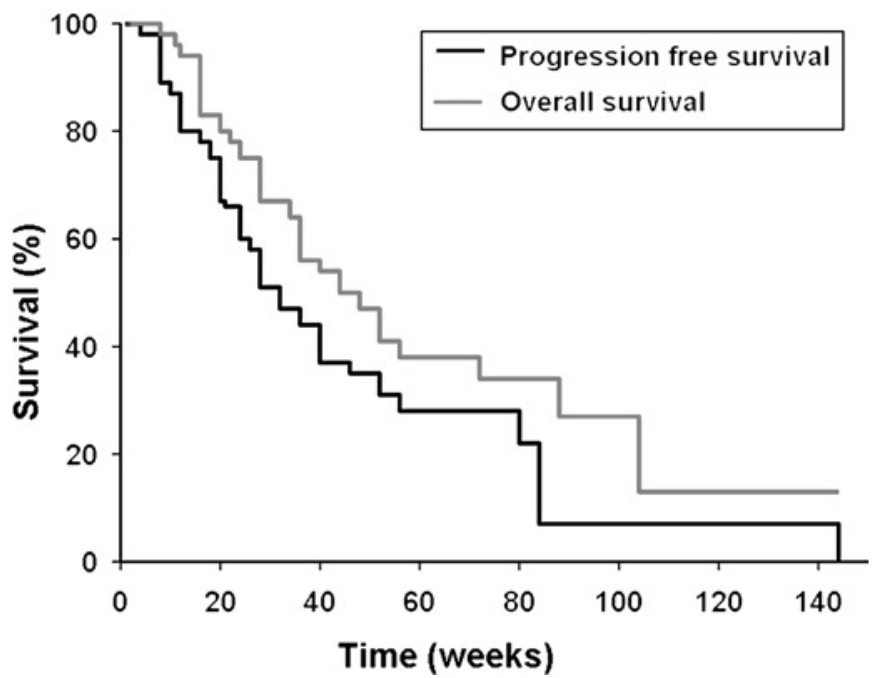

Figure 1 Kaplan-Meier estimates of progression-free survival and overall survival among patients with glioblastoma who were diagnosed by stereotactic biopsy and prospectively treated with radiotherapy plus concomitant and adjuvant temozolomide.

$\mathrm{p}=0.0008$ ); the prognostic influence of age (which was detected in one-variable models) was lost.

\section{DISCUSSION}

Currently, patients with non-resectable glioblastoma undergoing $\mathrm{XRT} / \mathrm{TMZ} \rightarrow \mathrm{TMZ}$ are left with uncertainties concerning their prognosis. Moreover, they are unlikely to be entered into randomised clinical trials, addressing molecular biomarkers such as MGMT methylation for patient stratification. Difficulties in performing molecular genetic analyses from small biopsy specimens have so far added to these barriers in knowledge and scientific evaluation. ${ }^{4}$ Here, we show in a prospective consecutive series of 56 non-resectable glioblastoma patients that by means of the still new technique of molecular stereotactic biopsy, even this somewhat neglected patient population can be addressed: overall survival for patients of this study cohort was similarly poor as compared with those undergoing biopsy only in the EORTC/NCIC trial. ${ }^{2}$ Outcome measurements, however, became highly divergent after stratification for $M G M T$ promoter methylation. Whereas patients harbouring a glioblastoma with an unmethylated $M G M T$ promoter experienced both extremely short PFS and OS, those with a methylated promoter exhibited surprisingly long PFS and OS. The time to progression was similar to that reported by Hegi et al ${ }^{4}$ after cytoreductive surgery
Figure 2 Kaplan-Meier estimates of progression-free survival $(A)$ and overall survival (B) among patients with glioblastoma stratified for the MGMT promoter methylation status who were diagnosed by stereotactic biopsy and prospectively treated with radiotherapy plus concomitant and adjuvant temozolomide. meth, methylated; unmeth, unmethylated. (a)

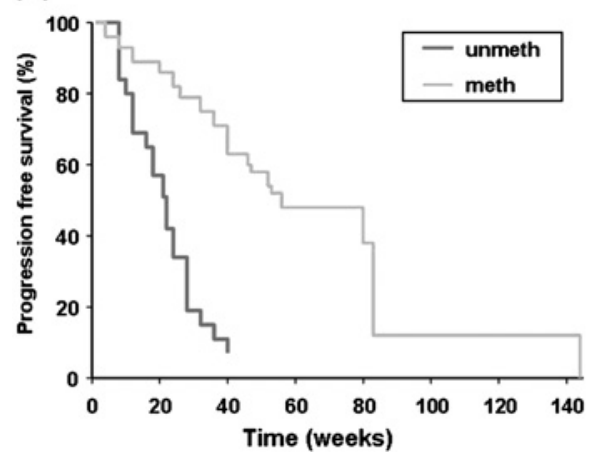

(b)

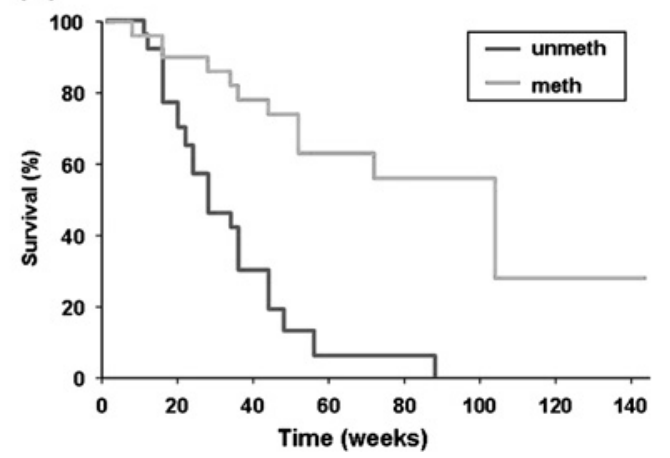


Table 3 Characteristics of patients with glioblastoma stratified for treatment response

\begin{tabular}{|c|c|c|c|}
\hline & $\begin{array}{l}\text { Non-responder } \\
\text { (progression) } \\
(\mathrm{N}=16)\end{array}$ & $\begin{array}{l}\text { Responder } \\
\text { (regressive }+ \\
\text { stable disease) } \\
(\mathrm{N}=40)\end{array}$ & p Value \\
\hline \multicolumn{4}{|l|}{ Characteristics } \\
\hline Median age (years) & 68.5 & 59.0 & 0.04 \\
\hline $\begin{array}{l}O^{6} \text {-methylguanine-DNA } \\
\text { methyltransferase methylated }\end{array}$ & $2(12.5 \%)$ & $28(70.0 \%)$ & 0.0008 \\
\hline $\begin{array}{l}0^{6} \text {-methylguanine-DNA } \\
\text { methyltransferase unmethylated }\end{array}$ & $14(87.5 \%)$ & $12(30.0 \%)$ & \\
\hline \multicolumn{4}{|l|}{ Outcome } \\
\hline Overall survival & & & $<0.0001$ \\
\hline Median (weeks) & 18 & 72 & \\
\hline Rate at 6 months & $37.5 \%$ & $90.0 \%$ & \\
\hline Rate at 12 months & $0 \%$ & $58.6 \%$ & \\
\hline
\end{tabular}

$\mathrm{N}$, number of patients.

plus $X R T / T M Z \rightarrow T M Z$. Survival after tumour progression, however, was shorter in the current series. One may speculate that the relatively large tumour volumes, which were not reduced by open surgery, have led to a relatively delayed diagnosis of tumour progression (using the Macdonald criteria). ${ }^{17}$ It should be further noted that a considerable number of patients received more than six cycles of TMZ in our series, which indicate an important difference to the EORTC/NCIC trial; accordingly, any comparative analysis should be avoided at this moment.

Early tumour progression was associated with poor outcome in all patients of this series. Apparently, pseudoprogression does not seem to be a frequent event in non-resectable glioblastoma patients undergoing XRT/TMZ. Partial response or tumour control (3 months after XRT/TMZ) was a strong predictor for a more favourable outcome and not unequivocally related to the MGMT promoter status: $93.3 \%$ of the MGMT methylated and, noteworthy, $46.2 \%$ of the unmethylated tumours exhibited partial response/stable disease after XRT/TMZ $\rightarrow$ TMZ. Survival, however, was significantly longer in case of a methylated MGMT promoter. Thus, for treatment responders with an unmethylated MGMT promoter, the benefit of XRT/ $\mathrm{TMZ} \rightarrow \mathrm{TMZ}$ as compared with XRT alone continues to be unclear. Future studies are required that further elucidate the molecular network of epigenetic silencing of the MGMT promoter and MGMT gene expression in correlation with clinical course and prognosis. ${ }^{23}$

Molecular stereotactic biopsy turned out to be a safe (transient morbidity of as low as $1.8 \%$ in this series) and precise diagnostic procedure. The highly controlled sampling technique ensured the use of only vital tumour tissues for subsequent molecular analyses, and excluded bias of the results by non-neoplastic tissue contaminations. Handling of nucleic acids as well as routine setup for MSP and sequencing analyses was successfully adapted to the small tissue samples containing limited amounts of nucleic acids. ${ }^{8}$ Due to these efforts, there were no drop-outs due to difficulties in determining the MGMT promoter status: the success rates of MSP and sequencing were $100 \%$, and both methods exhibited $100 \%$ concordant findings throughout each tumour investigated. The latter indicates a homogenous intratumoural distribution of the biomarker. The overall frequency of tumours harbouring a methylated MGMT promoter was $53.6 \%$, which was slightly higher than in the study by Hegi et al (MGMT promoter methylated tumours: $45 \%)^{4}$; the difference between these studies was not statistically significant and might have occurred by chance. However, the exact assignment of each biopsy specimen within the stereotactically defined tumour space, the determination of its relation to tumour necrosis or non-neoplastic tissue by alternating histopathological and molecular evaluation within $1 \mathrm{~mm}$ steps, the simultaneous application of two molecular genetic methods at different sites of each tumour and the exclusive use of cryopreserved specimens indicate important differences from the study by Hegi et al. ${ }^{4}$

We further demonstrate that the MGMT status after XRT/ $\mathrm{TMZ} \rightarrow \mathrm{TMZ}$ remained unchanged, which was seen in 13 patients undergoing stereotactic rebiopsy because of tumour progression. Both the detected intratumoural homogeneous distribution of the MGMT promoter methylation status and its unchanged pattern before and after XRT/TMZ $\rightarrow$ TMZ suggest that $M G M T$ promoter methylation may be a rather early event in tumour genesis.

In summary, the current series describes for the first time the powerful treatment effects of XRT/TMZ $\rightarrow \mathrm{TMZ}$ for patients with non-resectable glioblastoma in case of a methylated MGMT promoter. It underscores that non-resectable tumours should not be associated per se with a worse prognosis as compared with those undergoing open tumour resection. The demonstrated high diagnostic sensitivity and specificity of MSP

Table 4 Prognostic factors (univariate and multivariate models)

\begin{tabular}{lll}
\hline & $\mathbf{p}$ Value (hazard ratio/95\% CI) & \\
\cline { 2 - 3 } & Progression-free survival & Overall survival \\
\hline $\begin{array}{l}\text { Univariate } \\
0^{6} \text {-methylguanine-DNA } \\
\text { methyltransferase }\end{array}$ & $<0.0001(0.15 / 0.07$ to 0.33$)$ & $<0.0001(0.18 / 0.08$ to 0.38$)$ \\
$\begin{array}{l}\text { Age } \\
\text { Karnofsky performance score }\end{array}$ & $0.01(1.03 / 1.01$ to 1.06$)$ & $0.009(1.04 / 1.01$ to 1.08$)$ \\
$\begin{array}{l}\text { Radiation Therapy Oncology Group } \\
\text { Location of tumour }\end{array}$ & $0.01(0.95 / 0.92$ to 0.99$)$ & $0.003(0.94 / 0.90$ to 0.98$)$ \\
$\begin{array}{l}\text { Volume of tumour } \\
\text { Site of tumour }\end{array}$ & $0.04(1.57 / 1.02$ to 2.43$)$ & $0.01(1.85 / 1.13$ to 3.04$)$ \\
Multivariate & NS & NS \\
$\begin{array}{l}0^{6} \text {-methylguanine-DNA } \\
\text { methyltransferase }\end{array}$ & NS & NS \\
$\begin{array}{l}\text { Age } \\
\text { Karnofsky performance score }\end{array}$ & $<0.0001(0.16 / 0.07$ to 0.35$)$ & NS \\
\hline NS, not significant & $0.02(1.03 / 1.0$ to 1.06$)$ & $<0.0001(0.20 / 0.09$ to 0.44$)$ \\
& NS & NS \\
\hline
\end{tabular}

NS, not significant. 
and sequence analysis in combination with stereotactic biopsy technique might help to encourage the further development and implementation of molecular genetic analysis into biopsy procedures of daily clinical practice.

Acknowledgements M Ruiter is gratefully acknowledged for help with MSP and sequencing analysis. We further thank B Stetter, M Deschner and A Ewert for their support with management of the patient database.

Competing interests None.

Patient consent Obtained.

Ethics approval Ethics approval was provided by the German Glioma Network.

Provenance and peer review Not commissioned; externally peer reviewed.

\section{REFERENCES}

1. Wen PY, Kesari S. Malignant gliomas in adults. N Engl J Med 2008:359:492-507.

2. Stupp R, Hegi ME, Mason WP, et al. Effects of radiotherapy with concomitant and adjuvant temozolomide versus radiotherapy alone on survival in glioblastoma in a randomised phase III study: 5-year analysis of the EORTC-NCIC trial. Lancet Oncol 2009;10:459-66.

3. Stupp R, Mason WP, van den Bent MJ, et al. Radiotherapy plus concomitant and adjuvant temozolomide for glioblastoma. N Engl J Med 2005;352:987-96.

4. Hegi ME, Diserens AC, Gorlia T, et al. MGMT gene silencing and benefit from temozolomide in glioblastoma. N Engl J Med 2005:352:997-1003.

5. Gorlia T, van den Bent MJ, Hegi ME, et al. Nomograms for predicting survival of patients with newly diagnosed glioblastoma: prognostic factor analysis of EORTC and NCIC trial 26981-22981/CE.3. Lancet Oncol 2008:9:29-38.

6. Kreth S, Heyn J, Grau S, et al. Identification of valid endogenous control genes for determining gene expression in human glioma. Neuro-Oncology 2010;12:570-9.

7. Thon N, Eigenbrod S, Grasbon-Frodl EM, et al. Novel molecular stereotactic biopsy procedures reveal intratumoral homogeneity of loss of heterozygosity of $1 p / 19 q$ and TP53 mutations in World Health Organization Grade II Gliomas. J Neuropathol Exp Neurol 2009;68:1219-28.

8. Grasbon-Frodl EM, Kreth FW, Ruiter M, et al. Intratumoral homogeneity of MGMT promoter hypermethylation as demonstrated in serial stereotactic specimens from anaplastic astrocytomas and glioblastomas. Int $J$ Cancer 2007;121:2458-64.

9. Karnofsky DA, Abelmann WH, Craver LF, et al. The use of the nitrogen mustards in the palliative treatment of carcinoma. Cancer 1948;1:634-56.

10. Louis DN, Ohgaki H, Wiestler OD, et al. The 2007 WHO classification of tumours of the central nervous system. Acta Neuropathol 2007:114:97-109.

11. Kreth FW, Muacevic A, Medele R, et al. The risk of haemorrhage after image guided stereotactic biopsy of intra-axial brain tumours - a prospective study. Acta Neurochir (Wien) 2001:143:539-45.

12. Mollemann $\mathbf{M}$, Wolter $\mathbf{M}$, Felsberg J, et al. Frequent promoter hypermethylation and low expression of the MGMT gene in oligodendroglial tumors. Int J Cancer 2005:113:379-85.

13. Esteller M, Garcia-Foncillas J, Andion E, et al. Inactivation of the DNA-repair gene MGMT and the clinical response of gliomas to alkylating agents. N Engl J Med 2000:343:1350-4.

14. Clark SJ, Statham A, Stirzaker C, et al. DNA methylation: bisulphite modification and analysis. Nat Protoc 2006;1:2353-64.

15. Frommer $\mathbf{M}, \mathrm{McD}$ nald LE, Millar DS, et al. A genomic sequencing protocol that yields a positive display of 5-methylcytosine residues in individual DNA strands. Proc Natl Acad Sci U S A 1992:89:1827-31.

16. Curran WJ Jr, Scott CB, Horton J, et al. Recursive partitioning analysis of prognostic factors in three Radiation Therapy Oncology Group malignant glioma trials. J Natl Cancer Inst 1993:85:704-10.

17. Macdonald DR, Cascino TL, Schold SC Jr, et al. Response criteria for phase II studies of supratentorial malignant glioma. J Clin Oncol 1990;8:1277-80.

18. Brandes AA, Franceschi E, Tosoni A, et al. MGMT promoter methylation status can predict the incidence and outcome of pseudoprogression after concomitant radiochemotherapy in newly diagnosed glioblastoma patients. J Clin Oncol 2008;26:2192-7.

19. Popperl G, Kreth FW, Herms J, et al. Analysis of 18F-FET PET for grading of recurrent gliomas: is evaluation of uptake kinetics superior to standard methods? J Nucl Med 2006;47:393-403.

20. Chinot $\mathbf{O L}$, Barrie $\mathrm{M}$, Fuentes $\mathrm{S}$, et al. Correlation between $0^{6}$-methylguanine-DNA methyltransferase and survival in inoperable newly diagnosed glioblastoma patients treated with neoadjuvant temozolomide. J Clin Oncol 2007;25:1470-5.

21. Kaplan E, Meier P. Non parametric estimation for incomplete observation. J Am Stat Assoc 1958;53:457-81.

22. Cox DR. Regression model and life-tables. J Roy Stat Soc Ser B 1972:187-220.

23. Everhard S, Tost J, El AH, et al. Identification of regions correlating MGMT promoter methylation and gene expression in glioblastomas. Neuro Oncol 2009;11:348-56. 

in non-resectable glioblastoma after radiotherapy plus temozolomide

Niklas Thon, Sabina Eigenbrod, Eva M Grasbon-Frodl, Juergen Lutz, Simone Kreth, Gabriele Popperl, Claus Belka, Hans A Kretzschmar, Joerg-Christian Tonn and Friedrich W Kreth

J Neurol Neurosurg Psychiatry 2011 82: 441-446 originally published online September 22, 2010

doi: 10.1136/jnnp.2010.214593

Updated information and services can be found at:

http://jnnp.bmj.com/content/82/4/441

\section{These include:}

References This article cites 22 articles, 8 of which you can access for free at: http://jnnp.bmj.com/content/82/4/441\#BIBL
Email alerting service
Receive free email alerts when new articles cite this article. Sign up in the box at the top right corner of the online article.

Topic Articles on similar topics can be found in the following collections

Collections

CNS cancer (167)

Neurooncology (220)

Radiology (1606)

Surgical diagnostic tests (360)

Immunology (including allergy) (1706)

\section{Notes}

To request permissions go to:

http://group.bmj.com/group/rights-licensing/permissions

To order reprints go to:

http://journals.bmj.com/cgi/reprintform

To subscribe to BMJ go to:

http://group.bmj.com/subscribe/ 